\title{
Viscoelastic Properties of Poly(vinyl chloride) Gels: Effect of Plasticizer Type
}

\author{
Munetaka KAKIUCHI", Yuji AOKI*, Hiroshi WATANABE**, and Kunihiro OSAKI** \\ Chemical Science Laboratories, Mitsubishi Chemical Corporation, \\ 1, Toho-cho, Yokkaichi, Mie 510-8530, Japan* \\ Institute for Chemical Research, Kyoto University, Uji, Kyoto 611-0011, Japan ${ }^{* *}$ \\ (Received : September 1, 2000)
}

\begin{abstract}
Viscoelastic behavior of PVC gels/sols in a wide range of PVC concentration $c$ was examined in various plasticizers having different solubility toward PVC. The gel point $c_{\mathrm{g}}$ decreased with decreasing solubility, possibly due to enhanced formation of PVC crystallites (crosslinking points in the gels) in poorer plasticizers. The exponent characterizing the power-law behavior of the critical gel $\left(G^{\prime} \sim G^{\prime \prime} \sim \omega^{n}\right), n=0.75$, was insensitive to the plasticizer solubility. The exponent $z$ (=2.52 \pm 0.14$)$, characterizing the equilibrium modulus $G_{\mathrm{e}}$ of the well developed gels $\left(G_{\mathrm{e}} \sim \mathcal{E}^{z}\right.$ with $\left.\varepsilon=\left|c-c_{\mathrm{g}}\right| / c_{\mathrm{g}}\right)$ was also insensitive to the plasticizer solubility. However, the magnitude of the normalized modulus $c^{-1} G_{\mathrm{e}}$ was smaller in poor plasticizers, suggesting some heterogeneity in the gel network structure therein. The other exponent $\gamma$ characterizing the viscosity $\eta$ of the sols $\left(\eta \sim \varepsilon^{\prime}\right)$ moderately increases from 1.2 to 1.9 with increasing solubility. Comparison of the normalized viscosity and compliance in different plasticizers suggested that the sol structure was heterogeneous and this heterogeneity sensitively increased with decreasing solubility.
\end{abstract}

Key Words : Poly(vinyl chloride) /Plasticizer / Sol-gel transition / Physical gel

\section{INTRODUCTION}

Poly(vinyl chloride) (PVC) forms physical gels in various plasticizers, and their structures ${ }^{1 \sim 6)}$ and physical properties ${ }^{1,2}$, 5), 7 12) have been extensively investigated. The gels are generally classified as randomly crosslinked networks of flexible PVC strands, and the PVC crystal domains work as the crosslinking domains. Some properties of the gels such as the equilibrium elasticity are unequivocally related to this network structure. However, details of the structure of the crosslinking domains, either fibrous crystals ${ }^{1 \sim 3)}$ or small crystallites, ${ }^{6}$ have not been fully elucidated. In addition, the structures of the gel strands, either individual PVC chains or their fibrous aggregates, ${ }^{4), 5)}$ may change with temperature, concentration, solvent quality, and so on.

In attempt to rheologically investigate the sol/gel structure, $\mathrm{we}^{13-18)}$ recently began a series of studies for PVC's of various molecular weight $M_{\mathrm{w}}$ in bis(2-ethylhexyl) phthalate (DOP). At a constant temperature, $40^{\circ} \mathrm{C}$, the following scaling laws was found to hold below, at, and above the sol-gel transition concentration (gel point) $c_{\mathrm{g}} \cdot{ }^{13-15), 17)}$

$$
\begin{array}{lll}
\eta_{0} \propto \varepsilon^{-\gamma} & \text { for } & c<c_{\mathrm{g}} \\
G^{\prime}(\omega) \sim G^{\prime \prime}(\omega) \propto \omega^{n} & \text { for } & c=c_{\mathrm{g}}
\end{array}
$$

$$
G_{\mathrm{e}} \propto \varepsilon^{z} \quad \text { for } \quad c>c_{\mathrm{g}}
$$

Here, $\varepsilon\left(=\left|c-c_{\mathrm{g}}\right| / c_{\mathrm{g}}\right)$ is a relative distance to the gel point, $\eta_{0}$ is the zero shear viscosity, $G^{\prime}$ and $G^{\prime \prime}$ are the storage and loss moduli as a function of angular frequency $\omega$, and $G_{\mathrm{e}}$ the quasiequilibrium modulus. We found $M_{\mathrm{w}}$-insensitive exponent values, $n=0.75, \gamma=1.5$, and $z=2.6$. We also found a proportionality, $c_{\mathrm{g}} \propto M_{\mathrm{w}}{ }^{-1}$, which suggests an important role of the PVC chain size in formation of continuous gel networks.

In this paper, we extend our work to PVC gels/sols in several plasticizers having different solubilities toward PVC. We examine whether the scaling exponents are independent of the solubility of the plasticizers. The results are discussed in relation to the effect of solubility on the PVC sol/gel structure.

\section{EXPERIMENTS}

\subsection{Material}

A poly(vinyl chloride) (PVC), coded as PVC4 in the previous studies, ${ }^{13 \sim 18)}$ was used. This sample, previously synthesized via suspension polymerization at Mitsubishi Chemical Co., had a weight-average molecular weight $M_{\mathrm{w}}=$ $39.4 \times 10^{3}$, a heterogeneity index $M_{\mathrm{w}} / M_{\mathrm{n}}=1.9$, and a triad tacticity of syndio:hetero:iso $=0.33: 0.49: 0.18 .{ }^{13)}$

The systems subjected to rheological tests were gels/sols of 
Table I The molecular weights $M$, densities $\rho_{\mathrm{s}}$, and viscosities $\eta_{\mathrm{s}}$ of plasticizers

\begin{tabular}{cccccccc}
\hline plasticizer & DMP & DEP & DBP & DOP & DnOP & DUP & DOA \\
\hline$M(\mathrm{~g} / \mathrm{mol})$ & 194.2 & 222.2 & 278.3 & 390.6 & 390.6 & 474.7 & 370.6 \\
$\rho_{\mathrm{s}}\left(\mathrm{g} / \mathrm{cm}^{3}\right)$ & 1.190 & 1.118 & 1.043 & 0.981 & 0.972 & 0.954 & 0.925 \\
$\eta_{\mathrm{s}}(\mathrm{mPa} \mathrm{s})^{\mathrm{a}}$ & - & 6.2 & 7.6 & 26 & 15 & 22 & 6.8 \\
\hline
\end{tabular}

a) Measured at $40^{\circ} \mathrm{C}$.

PVC4 in several plasticizers. In the previous studies, bis(2ethylhexyl) phthalate (DOP) was used. ${ }^{13 \sim 18)}$ In this study, the other six plasticizers were also used; dimethyl phthalate (DMP), diehtyl phthalate (DEP), dibutyl phthalate (DBP), $n$ dioctyl phthalate (DnOP), diundecyl phthalate (DUP), and bis(2-ethylhexyl) adipate (DOA). These plasticizers have different solubilities toward PVC (as explained later for Fig.3), and some of their physical properties are summarized in Table I.

Prior to sample preparation, the PVC4 sample was purified by using tetrahydrofuran (THF)/methanol as the solvent/ precipitant. Six series of the PVC4/plasticizer systems were prepared at room temperature from mother solutions in THF (about $70 \mathrm{wt} \% \mathrm{THF}$ ) containing prescribed amounts of PVC and plasticizer. No stabilizer was added. The PVC concentration in each series was varied to cover the range from pregel (sol) state to the postgel state.

In the sample preparation, THF was allowed to evaporate from each mother solution at room temperature $\left(\cong 30^{\circ} \mathrm{C}\right)$ over a period of time $>2$ weeks. After this evaporation, transparent and viscoelastic PVC/plasticizer samples were recovered. Since the samples were equilibrated for sufficiently long times (including the THF evaporation time) prior to rheological measurements, respective samples exhibited no change in their properties with time.

\subsection{Measurements}

A rheometer (ARES; Rheometric Scientific) was employed to measure the storage shear modulus $G^{\prime}$ and the loss modulus $G^{\prime \prime}$ (in the linear viscoelastic regime) at $40^{\circ} \mathrm{C}$ as a function of angular frequency $\omega$. Parallel plate fixtures were used, and the plate diameter ( 25 or $50 \mathrm{~mm}$ ) was chosen according to the magnitude of the sample moduli. The sol/gel specimen was transferred from the flat laboratory dish into the rheometer fixture. Prior to each measurement, the specimen was annealed/stabilized at $40^{\circ} \mathrm{C}$ for $20 \mathrm{~min}$.

\section{RESULTS AND DISCUSSION}

3.1 Dynamic Viscoelastic Properties through the Sol-Gel Transition
Fig. 1 shows $G^{\prime}$ and $G^{\prime \prime}$ for the series of PVC4/DOA samples having the PVC concentrations $c$ as indicated. The liquid-like terminal behavior is observed for small $c$ :

$$
G^{\prime}(\omega) \propto \omega^{2} \quad G^{\prime \prime}(\omega) \propto \omega \quad(\text { at } \omega \rightarrow 0)
$$

Deviation from this behavior becomes pronounced with increasing $c$, and the solid-like behavior characterized by the $\omega$-insensitive $G^{\prime}$ is observed when $c$ is increased well beyond the gel point. This variation from the pregel state to the postgel state, observed for all PVC systems examined in this study, is very similar to that seen for the PVC/DOP systems. ${ }^{13)}$

\subsection{Determination of the Gel Point $c_{\mathrm{g}}$ and the Scaling Exponent $n$}

The scaling law at the gel point (Eq. (2)) allows us to accurately determine the gel point, $c_{\mathrm{g}}$. Eq. (2) is accompanied by a relationship that ensures the linear stimulus/response frame work,

$$
G^{\prime \prime}(\omega) / G^{\prime}(\omega)=\tan \delta=\tan (n \pi / 2)
$$

This frequency independence of loss tangent at $c=c_{\mathrm{g}}$, confirmed for various chemical and physical gels, ${ }^{8), 13), 17), 19 \sim 24)}$ has been used to determine the gel point.

Thus, for our PVC/plasticizer systems, we determined $c_{\mathrm{g}}$ from plots of $\tan \delta$ at various $\omega$ against the PVC concentration c. An example is shown in Fig.2 for the PVC4/DOA systems. All curves pass through the common point at $c=c_{\mathrm{g}}$.

The $c_{\mathrm{g}}$ 's of the PVC4 systems were accurately determined with the above method except for the PVC4/DMP system that had undetectably small $G^{\prime}$ for $c<c_{\mathrm{g}}$. The $c_{\mathrm{g}}$ of this PVC4/DMP system was less than $18.0 \mathrm{~g} / \mathrm{L}$ because $G^{\prime}$ exhibited the $\omega$ insensitive plateau at $c=18.0 \mathrm{~g} / \mathrm{L}$.

The values of $c_{\mathrm{g}}$ and the scaling exponent $n$ (Eqs. (2) and (5)) of the PVC4/systems are summarized in Table II. Clearly, $c_{\mathrm{g}}$ is dependent on but $n(=0.75 \pm 0.02)$ is independent of the plasticizer species. This $n$ value agrees with the value for the previously examined PVC4/DOP system. ${ }^{13), 17), 18)}$

Doty and Zable ${ }^{25)}$ conducted swelling experiments for crosslinked PVC and determined the interaction parameter $\chi$ 

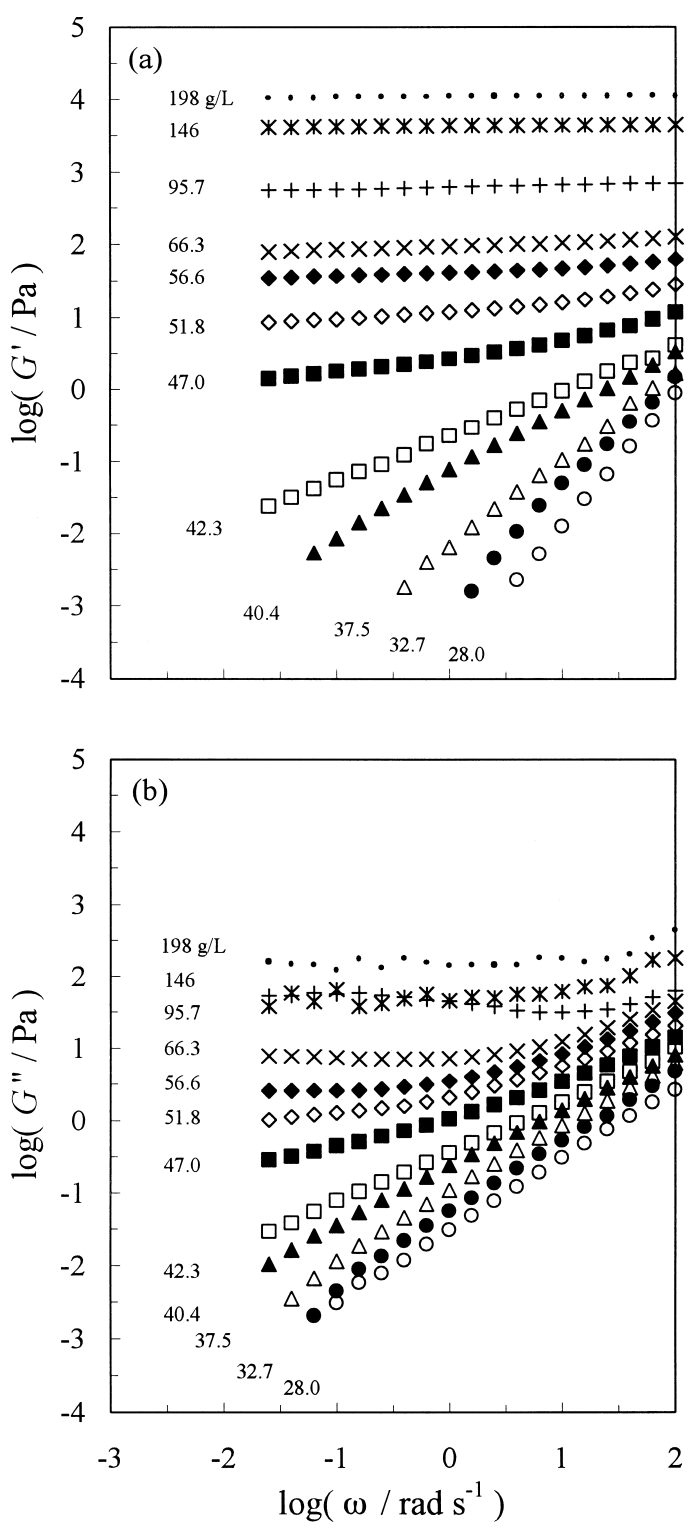

Fig.1 Plots of storage moduli $G^{\prime \prime}$ (a) and loss moduli $G^{\prime \prime}$ (b) against angular frequency $\omega$ for the PVC4/DOA system $\left(T=40^{\circ} \mathrm{C}\right)$ at various polymer concentration as indicated.

for many pairs of PVC and organic solvents. For a series of dialkyl phthalates having various molecular weights $M_{\mathrm{pl}}$ (i.e., various alkyl groups), they found that the $\chi$ is the smallest for $M_{\mathrm{pl}}=334$ (dihexyl phthalate). For $M_{\mathrm{pl}}<250$ and/or $M_{\mathrm{pl}}>450$, $\chi$ is larger than 0.5 (i.e., the plasticizer is a poor solvent for PVC). ${ }^{25)}$ Specifically, DEP and DMP are considerably poor solvents for PVC.

Table II The gel points $c_{\mathrm{g}}$, and scaling exponents $n, \gamma, z$ for various $\mathrm{PVC} /$ plasticizer systems

\begin{tabular}{cccccccc}
\hline plasticizer & DMP & DEP & DBP & DOP & DnOP & DUP & DOA \\
\hline$c_{\mathrm{g}}(\mathrm{g} / \mathrm{L})$ & $<18$ & 40.7 & 77.6 & 66.0 & 54.8 & 38.6 & 41.0 \\
$n$ & - & 0.75 & 0.76 & 0.75 & 0.76 & 0.77 & 0.76 \\
$\gamma$ & - & 1.10 & 1.73 & 1.54 & 1.36 & 1.18 & 1.09 \\
$z$ & - & 2.49 & 2.47 & 2.66 & 2.46 & 2.55 & 2.51 \\
\hline
\end{tabular}

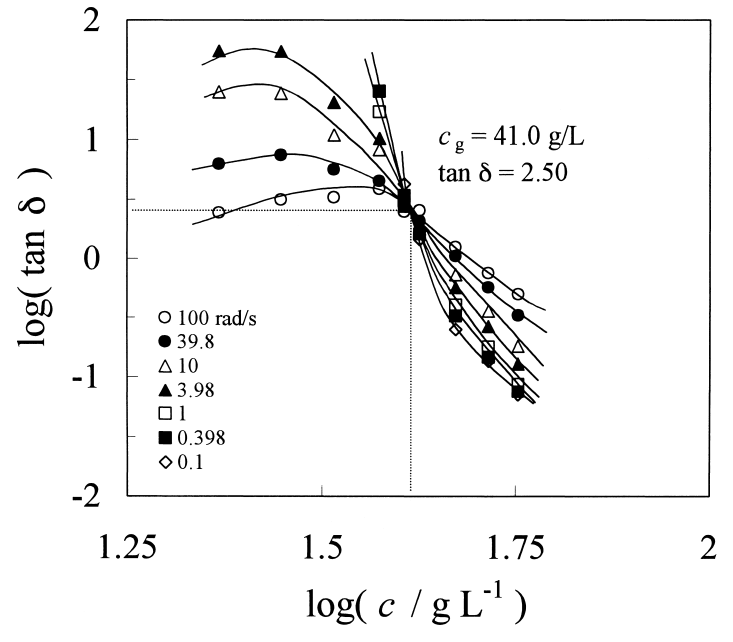

Fig.2 Plots of $\tan \delta$ against PVC concentration $c$ for the PVC4/DOA systems at various angular frequencies $(0.1,0.398,1,3.98,10$, $39.8,100 \mathrm{rad} / \mathrm{s}) . c_{\mathrm{g}}$ is the gel point.

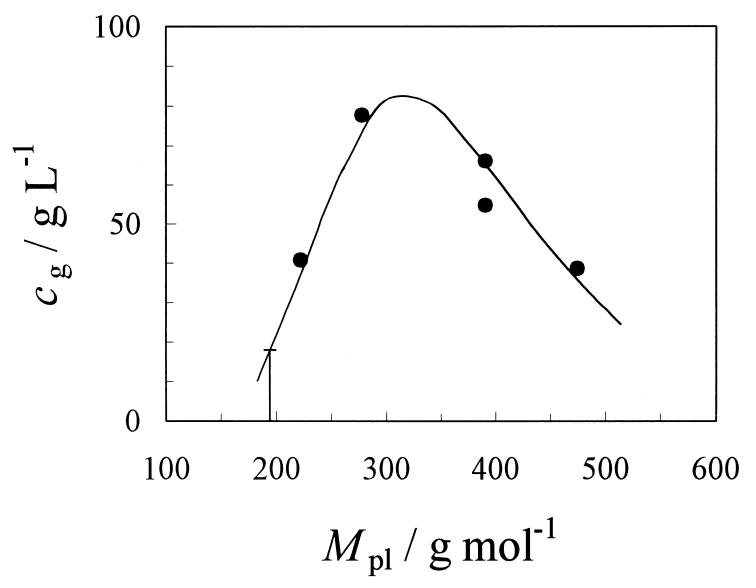

Fig.3 Variation of $c_{\mathrm{g}}$ with molecular weight of the dialkyl phthalate plasticizers.

The changes of the $c_{\mathrm{g}}$ of our PVC4 systems with the dilakyl phthalate species (Table II) are consistent with the above findings of Doty and Zable. As shown in Fig.3, $c_{\mathrm{g}}$ has the maximum value for $M_{\mathrm{pl}}$ between 278 and 391 and decreases with decreasing $M_{\mathrm{pl}}<278$ (i.e., for DEP and DMP) as well as with increasing $M_{\mathrm{pl}}>391$ (for DUP). Clearly, the gel is more easily formed in poorer solvents (plasticizers) possibly due to enhanced formation of PVC crystallites (crosslinking points in the gel). This effect of the plasticizer solubility on the gelation behavior becomes a key factor in our later discussion.

\subsection{Equilibrium Elasticity at $c>c_{\mathrm{g}}$}

Well developed gels exhibit the equilibrium modulus $G_{\mathrm{e}}(=$ $\left[G^{\prime}\right]_{\omega \rightarrow 0}$ ); see Fig.1(a). For those gels, Fig.4 shows plots of $G_{\mathrm{e}}$ against $c$. The $c$ dependence of $G_{\mathrm{e}}$ is different from system to system: The dependence is the strongest for the gels in DBP and DOP and weaker in the other plasticizers; the weakest 


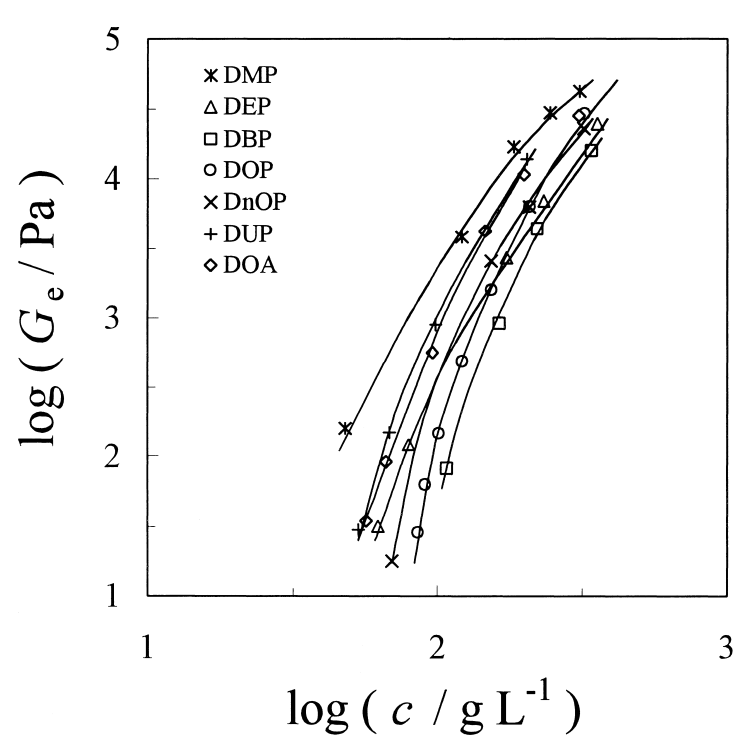

Fig.4 Plots of equilibrium modulus $G_{\mathrm{e}}$ of the PVC gels against PVC concentration $c$.

dependence is seen in DMP.

Dahmani et al. ${ }^{5)}$ observed similar differences of $G_{\mathrm{e}}$ for PVC gels in several plasticizers (diethyl oxalate, dibutyl oxalate, ethyl pelargonate, and ethyl valerate). They fitted their data with a power-law, $G_{\mathrm{e}} \propto c^{\alpha}$, and discussed the $\alpha$ values for respective gels in relation to the fractal dimension of a fragmented network in the corresponding sols. However, we should emphasize that our $G_{\mathrm{e}}$ data are not accurately described by this power-law; see Fig.4. Thus the concentration $c$ itself does not seem to be a quantity that determines the gel network structure.

From this point of view, we focus our attention to the relative distance from the gel point, $\varepsilon=\left(c-c_{\mathrm{g}}\right) / c_{\mathrm{g}}$. The $\varepsilon$ values of our gels were determined from the $c_{\mathrm{g}}$ data shown in Fig.3. Fig.5(a) shows plots of the $G_{\mathrm{e}}$ data of the gels against $\varepsilon$. (The data for the PVC/DMP gel are not shown because of the uncertainty in its $c_{\mathrm{g}}$ value.) In the entire range of $\varepsilon$ (and of $c$ ), the scaling (power-law) relationship, $G_{\mathrm{e}} \propto \mathcal{E}^{z}$ (Eq. (3)), holds within experimental uncertainties, and the gels in various plasticizers have an almost identical exponent $z(=2.52 \pm$ $0.14)$; cf. Table II. This identity of $z$ suggests the similarity of the network structure in those gels at a given $\varepsilon$. Thus the differences of the $c$ dependence of $G_{\mathrm{e}}$ seen in Fig.4 is mainly related to the difference of $c_{\mathrm{g}}$ in various plasticizers.

However, Fig.5(a) also demonstrates that the $G_{\mathrm{e}}$ values are different for the gels in various plasticizers at a given $\varepsilon$. This result reflects a fact that $G_{\mathrm{e}}$ of a polymeric system is generally proportional to a factor specifying the structure therein as well as to the polymer mass per unit volume, i.e. to $c$. A good example of this fact is found for the entanglement plateau
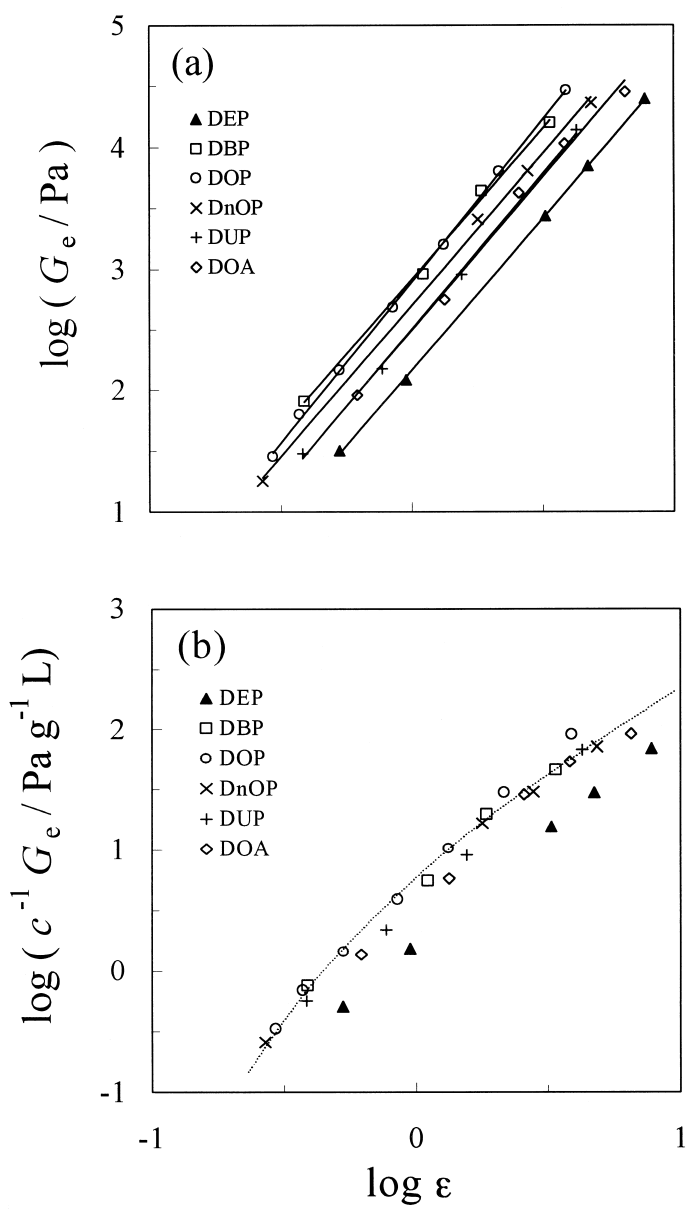

Fig.5 (a) Equilibrium modulus $G_{\mathrm{e}}$ of the PVC gels plotted against the relative distance to the gel point, $\varepsilon=\left|c-c_{\mathrm{g}}\right| / c_{\mathrm{g}}$. (b) Plots of the normalized modulus $G / c$ against $\varepsilon$.

modulus $G_{\mathrm{N}}$ of usual polymer solutions (not gelling systems). Experiments indicate a power-law relationship, ${ }^{26)} G_{\mathrm{N}} \propto c / M_{\mathrm{e}} \propto$ $c^{1+d}\left(M_{\mathrm{e}} \propto c^{-d}\right)$ with $d=1-1.3$, where the entanglement molecular weight $M_{\mathrm{e}}$ is the quantity characterizing the entanglement mesh structure. Thus, this mesh structure is most clearly examined for $G_{\mathrm{N}} / c$, not $G_{N}$ itself.

The same concept applies also to the gels. Namely, the gel network structure is most straightforwardly reflected in $G_{\mathrm{e}} / c$, not in $G_{\mathrm{e}}$ itself. Considering this fact, we examine the $\varepsilon$ dependence of $G_{\mathrm{e}} / c$ in Fig.5(b). The scattering of the plots for the gels in various plasticizers is smaller in Fig.5(b) (see the collapse around the dotted curve) than in Fig. 5(a), except for the gel in $\operatorname{DEP}(\boldsymbol{\Delta})$. Thus, the $G_{\mathrm{e}} / c$ data are almost identical, in both magnitude and $\varepsilon$ dependence, for the gels in various plasticizers (except in DEP), and this dependence becomes weaker with increasing $\varepsilon$ commonly for these gels. This result confirms that the gels include almost identical network structure determined by $\varepsilon$.

Concerning this result, we have to add a few comments for the scaling relationship, Eq. (3). Theoretically, this relationship 
is derived for critical phenomena and is more accurately valid for $\varepsilon \rightarrow 0$, i.e., on approach of $c$ toward $c_{\mathrm{g}}$. In the real close vicinity of $c_{\mathrm{g}}$, a very small increase of $c$ results in large increases of $\varepsilon$ and $G_{\mathrm{e}}$. In this vicinity, the polymer mass per unit volume (contributing linearly to $G_{\mathrm{e}}$; see the above argument) is almost constant and we can simply examine the gel network structure through the $\varepsilon$ dependence of $G_{\mathrm{e}}$. However, in a wider range of $c$ (as in our experiment that covers $\varepsilon$ between 0.3 and 10 ), we have to consider the change of this polymer mass and normalize $G_{\mathrm{e}}$ by $c$. The universal $\varepsilon$ dependence of $G / c$ of various gels, seen in Fig.5(b) (except for the gel in DEP), certainly suggests the similarity of the gel network structure in those gels at a given $\varepsilon$, although the gels at large $\varepsilon$ are out of the real critical regime. In other words, the gel network structure appears to be uniquely determined by $\varepsilon$ in both critical and out-critical regimes. (In Fig.5(b), the weakening of the $\varepsilon$ dependence of $G_{\mathrm{e}} / c$ at large $\varepsilon$ reflects a crossover from the critical to out-critical regime.)

Finally, we discuss the breakdown of the universality for the gel in DEP ( $\boldsymbol{\Delta}$ in Fig.5(b)). DEP is a considerably poor solvent for PVC (as explained for Fig.3). ${ }^{5)}$ Thus, some heterogeneity in the PVC concentration distribution (prephase-separation effect) would exist in the PVC sol in DEP and this heterogeneity could survive to some extent in the PVC/DEP gel. The difference of $G_{\mathrm{e}} / c$ of this gel may be related to this surviving heterogeneity.

\subsection{Viscosity and Compliance at $c<c_{g}$}

For the PVC sols, we here attempt to examine the sol structure through the zero-shear viscosity $\eta_{0}$. For this purpose, we first need to specify the relationship between $\eta_{0}$ and structure, as we did for $G_{\mathrm{e}}$.

In general, the polymer contribution to $\eta_{0}$ is proportional to a structural factor $F$ as well as to the polymer mass per unit volume (c) and the monomeric friction $\zeta .{ }^{26)}$ The $F$, the quantity of our interest, changes with various factors such as $M_{\mathrm{w}}$ and degree of branching of individual chains, a degree of mutual overlapping of chains, and the fragmented network structure physically crosslinked at crystal domains (in the case of the PVC gels). In contrast, $\zeta$ simply changes with the solvent viscosity $\eta_{\mathrm{s}}$ and $c$ : For rather dilute PVC sols, the change of $\zeta$ with $c$ is minor and we can approximate that $\zeta$ is proportional to $\eta_{\mathrm{s}}$.

Considering these points, we have examined the $c$ and $\varepsilon$ dependencies of two types of reduced viscosities of our PVC sols, $\eta_{\mathrm{r}}=\left(\eta_{0}-\eta_{\mathrm{s}}\right) / \eta_{\mathrm{s}}$ and $\eta_{\mathrm{r}} / c: \eta_{\mathrm{r}}$ is a quantity corrected for the solvent contribution and the $\zeta$ factor explained above, and $\eta_{\mathrm{r}} / c$
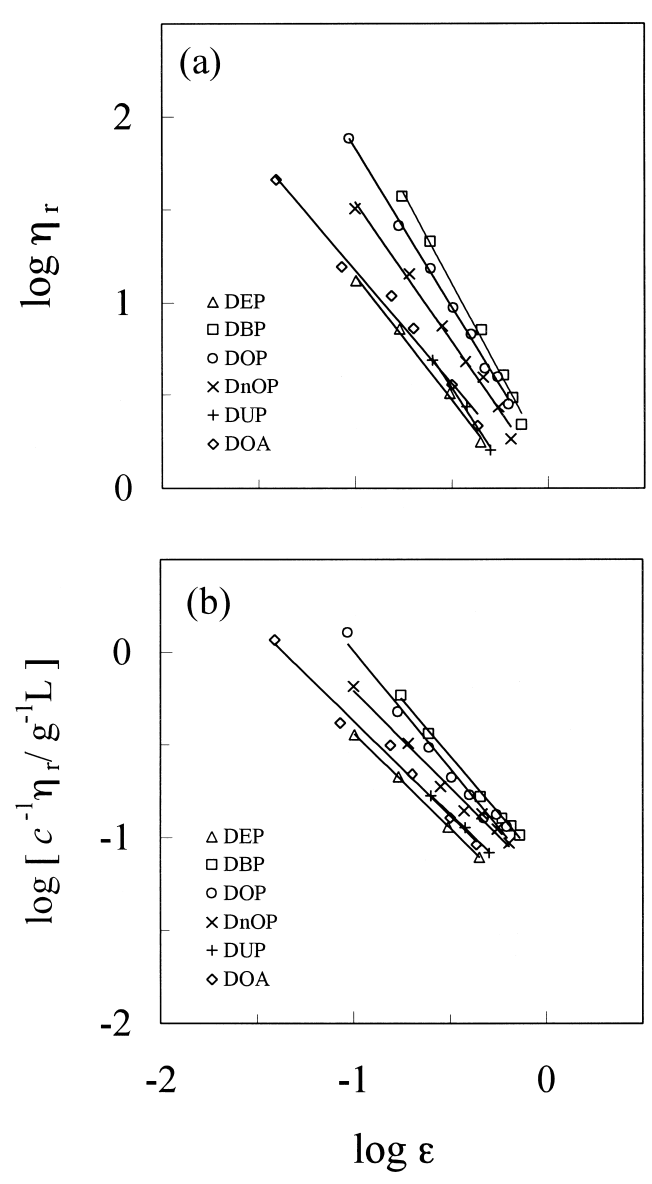

Fig.6 (a) Relative viscosity $\eta_{\mathrm{r}}$ plotted against the relative distance to the gel point, $\varepsilon=\left|c-c_{\mathrm{g}}\right| / c_{\mathrm{g}}$. (b) Plots of the normalized viscosity $\eta_{\mathrm{r}} /$ $c$ against $\varepsilon$.

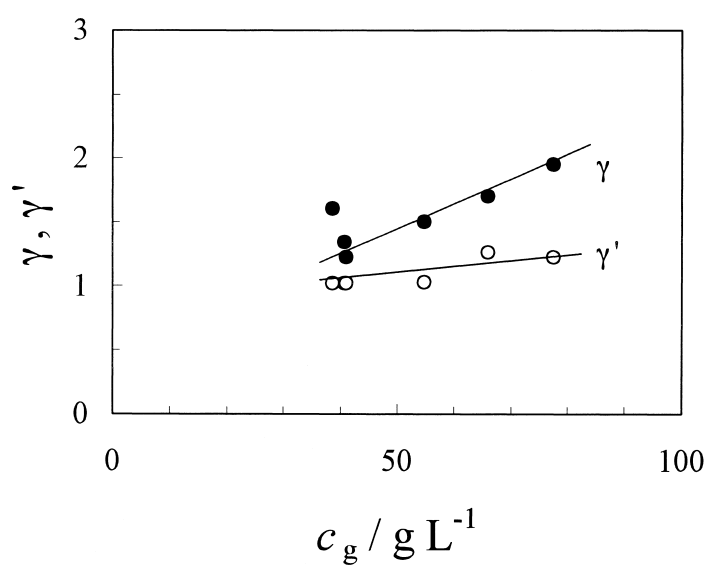

Fig.7 Plots of scaling exponents $\gamma$ and $\gamma^{\prime}$ of sols against $c_{\mathrm{g}}$.

is further corrected for the polymer mass per unit volume (and reflects $F$ straightforwardly). We found that neither $\eta_{\mathrm{r}}$ nor $\eta_{\mathrm{r}} / c$ of the sols in various plasticizers exhibit the universal $c$ dependence. This result, analogous to the difference of $G_{\mathrm{e}}$ of various gels (Fig.4), indicates that the sol structure is not determined by $c$ itself. In contrast, interesting dependencies on $\varepsilon$ are found for both $\eta_{\mathrm{r}}$ and $\eta_{\mathrm{r}} / c$, as demonstrated in Fig.6.

Fig.6(a) indicates that a scaling relationship, $\eta_{\mathrm{r}} \cong \eta_{0} / \eta_{\mathrm{s}} \propto \mathcal{E}^{\gamma}$ 
(equivalent to Eq. (1)), is valid within experimental uncertainty for the sols examined. The exponent $\gamma$ increases with increasing solubility of the plasticizer toward PVC and thus with increasing $c_{\mathrm{g}}$, as shown in Fig.7. This increase of $\gamma$ may be related the fragmented network (PVC clusters) in the sols that becomes more compact to have larger fractal dimension in worse solvents. However, the $\varepsilon$ range examined $(0.1<\varepsilon<1$ for most sols) does not necessarily coincide with the real critical regime $(\varepsilon<<1)$ where the gelation can be regarded as the critical phenomenon (see the argument for $G_{\mathrm{e}}$ ), and the increase of $\eta_{\mathrm{r}}$ with decreasing $\varepsilon$ seen in Fig.6(a) may be partly contributed from the increase of the polymer mass; remember that $\eta_{0}-\eta_{\mathrm{s}} \propto \zeta_{c F}\left(\propto \eta_{\mathrm{s}} c F\right.$ for rather dilute sols $)$. The importance of this contribution can be examined for $\eta_{\mathrm{r}} / c$ (Fig.6(b)).

Fig.6(b) demonstrates the power-law relationship, $\eta_{\mathrm{r}} / c \propto \mathcal{E}^{\gamma^{\prime}}$. The exponent $\gamma^{\prime}$ hardly change with the solubility of the plasticizer, as shown in Fig.7. Thus, the compactness of the PVC sol cluster cannot be deduced from this exponent $\gamma^{\prime}$, and the increase of $\gamma$ (defined for $\eta_{\mathrm{r}}$; filled circles) may be significantly contributed from the increase of the polymer mass.

However, Fig.6(b) also demonstrates that $\eta_{\mathrm{r}} / c$ decreases systematically with decreasing solubility of the plasticizer. This result does suggest that the clusters of the PVC chains in the sol are more compact and densely branched in poorer plasticizers.

In further discussion of this difference of the sol cluster structure, we can examine a normalized compliance, $c J_{\text {pol }}$, where $J_{\text {pol }}=\left[G^{\prime} /\left(G^{\prime \prime}-\omega \eta_{\mathrm{s}}\right)^{2}\right]_{\omega \rightarrow 0}$ is the steady state compliance of the PVC chains in the sol. Analogously to $G_{\mathrm{N}}$ of the gels, $J_{\text {pol }}$ of the sol reflects the sol cluster structure $(F)$ and is inversely proportional to the polymer mass per unit volume $(c)$ but independent of $\zeta{ }^{26)}$, 27) Thus the sol structure and its heterogeneity are represented straightforwardly by $c J_{\text {pol }}$.

Fig. 8 compares $c J_{\text {pol }}$ of the sols in two poor plasticizers (DEP and DOA) and two better plasticizers (DBP and DOP). A significant difference of $c J_{\text {pol }}$ is observed for the two classes of the plasticizers. This difference unequivocally indicates changes of the sol cluster structure with the plasticizer solubility. Concerning this point, we also note that the difference of $c J_{\text {pol }}$ of the sols in DBP and DOP (two better plasticizers) is considerably larger than the difference of $G_{\mathrm{e}} / c$ of the gels in the same solvents (cf. Figs.5(b) and 8). This result might mean that the heterogeneity in the sol cluster structure changes sensitively with the plasticizer solubility but this heterogeneity decreases with increasing $c$ above $c_{\mathrm{g}}$. A

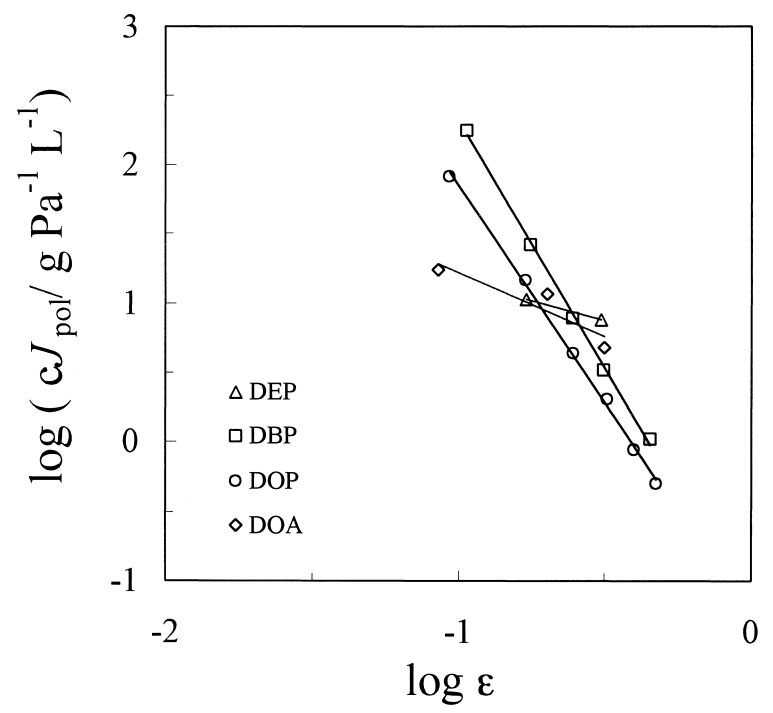

Fig.8 Plots of the normalized compliance $c J_{\text {pol }}$ against $\varepsilon$.

further study is desirable for this structural change with $c$.

\section{CONCLUDING REMARKS}

We have investigated viscoelastic behavior of PVC gels/sols in various plasticizers having different solubility toward PVC. The gel point $c_{\mathrm{g}}$ decreased with decreasing solubility possibly due to enhanced formation of PVC crystallites (crosslinking points in the gels) in poorer plasticizers. The exponent $n$ characterizing $G^{\prime}$ and $G^{\prime \prime}$ of the critical gel $\left(G^{\prime} \sim G^{\prime \prime} \sim \omega^{n}\right)$ was insensitive to the plasticizer solubility and had a value, $n=$ 0.75 . The exponent $z(=2.52 \pm 0.14)$, characterizing the equilibrium modulus of the well developed gels $\left(G_{\mathrm{e}} \sim \mathcal{E}^{z}\right)$, was also insensitive to the solubility. However, the magnitude of the normalized modulus $c^{-1} G_{\mathrm{e}}$ was smaller in the poor plasticizer (DEP), suggesting some heterogeneity in the gel network structure therein. The other exponent $\gamma$ characterizing the viscosity of sols $\left(\eta_{\mathrm{r}} \sim \mathcal{E}^{\gamma}\right)$ moderately increases from 1.2 to 1.9 with increasing solubility. Comparison of the normalized viscosity and compliance in different plasticizers suggested that the sol structure is heterogeneous and this heterogeneity sensitively increases with decreasing solubility.

\section{References}

1) Mutin PH, Guenet JM, Macromolecules, 22, 843 (1989).

2) Najeh M, Munch JP, Guenet JM, Macromolecules, 25, 7018 (1992).

3) Reinecke H, Saiani A, Mijamgous C, Guenet JM, Macromolecules, 29, 4799 (1996).

4) Reinecke $\mathrm{H}$, Mijamgous $\mathrm{C}$, Brulet A, Guenet JM, Macromolecules, 30, 959 (1997). 
5) Dahmani M, Fazel N, Munch JP, Guenet JM, Macromolecules, 30, 1463 (1997).

6) Keller A, Faraday Discuss, 101, 1 (1995).

7) Takahashi A, Nakamura T, Kagawa I, Polymer J, 3, 207 (1972).

8) Nijenhuis K, Winter HH, Macromolecules, 22, 411 (1989).

9) Garcia A, Munoz ME, Pena J J, Santamaria A, Macromolecules, 23, 5251 (1990).

10) Mijangos C, Lopez D, Munoz ME, Santamaria A, Macromolecules, 26, 5693 (1993).

11) Lopez D, Mijangos C, Munoz ME, Santamaria A, Macromolecules, 29, 7108 (1996).

12) Nijenhuis K, Adv. Polm. Sci., 130, 1 (1997).

13) Li L, Aoki Y, Macromolecules, 30, 7835 (1997).

14) Li L, Uchida H, Aoki Y, Yao M, Macromolecules, 30, 7842 (1997).

15) Li L, Aoki Y, Macromolecules, 31, 740 (1998).

16) Watanabe H, Sato T, Osaki K, Aoki Y, Li L, Kakiuchi M, Yao M, Macromolecules, 31, 4198 (1998).
17) Aoki $Y$, Li L, Uchida H, Kakiuchi M, Watanabe H, Macromolecules, 31, 7472 (1998).

18) Aoki Y, Li L, Kakiuchi M, Macromolecules, 31, 8117 (1998).

19) Winter HH, Chambon F, J Rheol, 30, 367 (1986).

20) Scanlan J C, Winter HH, Macromolecules, 24, 47 (1991).

21) Izuka A, Winter HH, Hashimoto T, Macromolecules, 25, 2422 (1992).

22) Koike A, Nemoto N, Takahashi M, Osaki K, Polymer, 35, 3005 (1994).

23) Takahashi M, Yokoyama K, Masuda T, Takigawa T, J Chem Phys, 101, 798 (1994).

24) Koike A, Nemoto N, Watanabe Y, Osaki K, Polym J, 28, 942 (1996).

25) Doty P, Zable HS, J Polym Sci, 1, 90 (1946).

26) Ferry JD, "Viscoelastic Properties of Polymers", 3rd ed, (1980), John Wiley \& Sons Inc, NY.

27) Watanabe H, Osaki K, Kakiuchi M, Aoki Y, Macromolecules, in press (2000). 